\title{
Evaluación funcional y del dolor en pacientes con deformidad espinal manejados quirúrgicamente
}

\author{
Functional and pain evaluation in patients with spinal deformity surgically treated
}

\author{
Arturo García-Galicia', José A. Vázquez-Roblero, Edgar Corpus-Mariscal', José L. García-Navarro', \\ Álvaro J. Montiel-Jarquín ${ }^{*}$ y Jorge Loría-Castellanos ${ }^{3}$ \\ 'Unidad Médica de Alta Especialidad, Hospital de Traumatología y Ortopedia de Puebla, Centro Médico Nacional Gral. de Div. Manuel Ávila

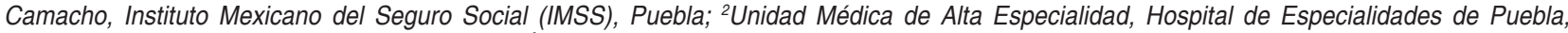 \\ Centro Médico Nacional Gral. de Div. Manuel Ávila Camacho, IMSS, Puebla; ${ }^{3}$ División de Programas Especiales en Salud, IMSS, Ciudad de \\ México. México
}

\section{Resumen}

Antecedentes: La columna vertebral degenerativa tiene una incidencia $>60 \%$ en los mayores de 60 años. La funcionalidad y el dolor se evalúan con el Oswestry Disability Index (ODI) y la Escala Visual Análoga (EVA), respectivamente. Objetivo: Evaluar la funcionalidad y el dolor en pacientes con columna vertebral degenerativa a 1 año de operados. Método: Estudio comparativo y longitudinal de pacientes con columna vertebral degenerativa manejados quirúrgicamente durante 2016 a 2018, a quienes se evaluaron aspectos funcionales y el dolor antes de la cirugía y 1 año después mediante ODI y EVA. Se utilizaron estadística descriptiva, medidas de tendencia central y dispersión, y prueba $t$ de Student para la diferencia entre los valores preoperatorios y posoperatorios de ambas escalas. Resultados: De 18 pacientes, 15 completaron seguimiento (8 hombres y 7 mujeres). La edad promedio fue de $63.5 \pm 4.8$ años. Trece presentaron limitación funcional posquirúrgica moderada y dos limitación funcional intensa. Hubo diferencias estadísticamente significativas entre el preoperatorio y el posoperatorio para el ODI y la EVA ( $p=0.011$ y $p=0.017$, respectivamente). Conclusiones: La funcionalidad y el dolor evaluados 1 año después de la cirugía tienen diferencia estadísticamente significativa en comparación con las evaluaciones antes de la cirugía.

Palabras clave: Columna vertebral degenerativa. Deformidad espinal. Dolor. Limitación funcional. Oswestry disability index.

\begin{abstract}
Background: The degenerative spine has an incidence greater than $60 \%$ in people over 60 years old. Functionality and pain is assessed by the Oswestry Disability Index (ODI) and the visual analogue pain scale (VAPS), respectively. Objective: To evaluate the functionality and pain one year after surgery, in patients with degenerative spine. Method: Comparative, longitudinal study, in patients with degenerative spine surgically managed during 2016 to 2018. Functional aspects and pain were evaluated before surgery and one year later using ODI and VAPS. Descriptive statistics, measures of central tendency and dispersion, Student's $t$ were used for the difference between the pre and postoperative values of both scales. Results: There were 18 patients. Of these, 15 completed the follow-up; 8 were men and 7 women. The average age was $63.5 \pm 4.8$ years; 13 presented moderate post-surgical functional limitation, 2 intense functional limitation. There were statistically significant
\end{abstract}

\footnotetext{
Correspondencia:

*Álvaro J. Montiel-Jarquín

Calle 2 Norte 2004

Col. Centro

Fecha de recepción: 23-09-2019

C.P. 72000 , Puebla, Pue., México

E-mail: alvaro.montielj@imss.gob.mx; dralmoja@ hotmail.com

Fecha de aceptación: 18-05-2020

DOI: $10.24875 / \mathrm{CIRU} .20001585$

0009-7411/@ 2020 Academia Mexicana de Cirugía. Publicado por Permanyer. Este es un artículo open access bajo la licencia CC BY-NC-ND (http://creativecommons.org/licenses/by-nc-nd/4.0/).
} 
differences between the pre and postoperative values for ODI and VAPS ( $p=0.011$ and $p=0.017$, respectively). Conclusions: Functionality and pain evaluated one year after surgery, have a statistically significant difference compared to evaluations before surgery in patients with spine deformity surgically treated.

Key words: Degenerative spine. Spinal deformity. Pain. Functional limitation. Oswestry disability index.

\section{Introducción}

La columna vertebral degenerativa del adulto es un trastorno espinal desafiante para el médico tratante'. La decisión entre realizar un tratamiento médico o quirúrgico en ocasiones en difícil, y radica en factores como la gravedad de los síntomas del paciente, el riesgo de la intervención y las condiciones propias del paciente ${ }^{2-4}$.

Dentro de las técnicas quirúrgicas descritas, la fijación posterior larga hacia la región torácica superior reduce la incidencia de cirugía de revisión, pero aumenta la complejidad y la cantidad de sangrado, y prolonga el tiempo quirúrgico $0^{5,6}$. A pesar de que los resultados quirúrgicos son alentadores, en ocasiones no todos los pacientes evolucionan adecuadamente. Los resultados dependen de factores asociados al paciente, como el índice de masa corporal, el estado de depresión/ansiedad, el tabaquismo, la gravedad del dolor, la presencia de comorbilidad y la gravedad de la deformidad ${ }^{7,8}$, así como de factores inherentes al cirujano que realiza el procedimiento, como su habilidad y experiencia. Los pacientes mayores, con comorbilidad como diabetes mellitus e hipertensión arterial sistémica, tienen una probabilidad mayor de evolucionar de manera inadecuada ${ }^{7,9,10}$; la tasa de complicaciones aumenta al $71 \%$ en los pacientes mayores de 65 años ${ }^{7}$.

Los pacientes con deformidad espinal degenerativa suelen reportar una mejoría significativa a los 2 años de seguimiento ${ }^{7}$.

El Oswestry Disability Index (ODI) evalúa la limitación funcional permanente del paciente con patología de columna vertebral y se considera el método de referencia para el diagnóstico de funcionalidad de la espalda baja. Evalúa 10 actividades cotidianas con una puntuación subjetiva de la función, es autocompletado y de aplicación fácil, ya que puede ser realizado por vía telefónica'11. Evalúa la función, el dolor y la limitación en la salud ${ }^{12,13}$. Esta escala, en su versión en español latinoamericano, ya ha sido utilizada en estudios en Latinoamérica con resultados clinimétricos satisfactorios ${ }^{14,15}$.
Para evaluar el dolor, la escala visual análoga (EVA) del dolor es una forma fácil, sencilla y eficaz para determinar el grado de dolor presentado por el paciente a cualquier nivel'

El objetivo de este trabajo de investigación fue evaluar la funcionalidad y el dolor en pacientes con columna vertebral degenerativa a 1 año de operados.

\section{Método}

Estudio comparativo y longitudinal en pacientes con deformidad espinal atendidos quirúrgicamente a quienes se evaluó la funcionalidad y el dolor antes de la cirugía y 1 año después. Se incluyeron pacientes de ambos sexos, con deformidad espinal degenerativa, manejados quirúrgicamente, que aceptaron participar en el estudio mediante el llenado de un consentimiento informado. Se excluyeron los pacientes con cáncer o con fracturas traumáticas o patológicas en la columna, y aquellos con patología de columna independiente posterior a la atención quirúrgica primaria, y se eliminaron los que no completaron los cuestionarios de evaluación al $100 \%$. Se realizó un muestreo consecutivo conveniente y se reclutó a todos los pacientes que cumplieron con los criterios de selección atendidos en el Hospital de Traumatología y Ortopedia de Puebla de 2016 a 2018.

La evaluación de la funcionalidad fue a través de la ODI, versión para población mexicana, y la del dolor con la EVA, a 12 meses de realizada la cirugía. Ambas escalas han sido ampliamente validadas y utilizadas en todo el mundo ${ }^{16}$. Las variables utilizadas fueron la edad, el sexo, el diagnóstico, la comorbilidad, el tiempo y el sangrado transquirúrgicos, las complicaciones y la estancia hospitalaria. El tipo de cirugía fue de acuerdo con la clasificación de Schwab ${ }^{17}$.

Este estudio fue aprobado por los comités de ética y de investigación correspondientes.

Se utilizaron estadística descriptiva, medidas de tendencia central y dispersión, para demostrar las diferencias entre las evaluaciones preoperatoria y posoperatoria, con la prueba t de Student, en el programa estadístico SPSS v. 23 para Windows. 
Tabla 1. Características de los 15 pacientes incluidos en el estudio

\begin{tabular}{lcc}
\hline \multicolumn{3}{c}{ Sexo } \\
\hline Mujer $\mathbf{n}(\%)$ & Hombre $\mathbf{n}(\%)$ \\
\hline $8(53)$ & $7(47)$ \\
\hline \multicolumn{3}{c}{ Diagnóstico } \\
\hline $\begin{array}{l}\text { Escoliosis degenerativa } \\
\text { del adulto } \mathbf{n}(\%)\end{array}$ & $\begin{array}{c}\text { Espondilolistesis } \\
\mathbf{n}(\%)\end{array}$ & $\begin{array}{c}\text { Cifosis } \\
\mathbf{n}(\%)\end{array}$ \\
\hline $9(60)$ & $5(33.3)$ & $1(6.6)$ \\
\hline \multicolumn{3}{c}{ Comorbilidad } \\
\hline $\begin{array}{l}\text { Diabetes mellitus } \\
\text { tipo 2 } \mathbf{n}(\%)\end{array}$ & Hipertensión arterial & $\begin{array}{c}\text { Cardiopatía } \\
\mathbf{n}(\%)\end{array}$ \\
\hline $3(37.5)$ & $4(50)$ & $1(6.66)$ \\
\hline
\end{tabular}

Tabla 2. Características de las cirugías realizadas en los pacientes

\begin{tabular}{|c|c|c|c|}
\hline \multicolumn{4}{|c|}{ Tiempo quirúrgico (min) } \\
\hline Media & Mínimo & Máximo & DE \\
\hline 325 & 180 & 480 & 95 \\
\hline \multicolumn{4}{|c|}{ Sangrado (ml) } \\
\hline Media & Mínimo & Máximo & DE \\
\hline 1940 & 1000 & 3500 & 841 \\
\hline \multicolumn{4}{|c|}{ Técnica de osteotomía* } \\
\hline $\begin{array}{l}\text { Tipo II: resección } \\
\text { completa de } \\
\text { articulaciones } \\
\text { facetarias }\end{array}$ & \multicolumn{2}{|c|}{$\begin{array}{c}\text { Tipo IV: sustracción } \\
\text { pedicular/discal parcial/ } \\
\text { cuerpo vertebral }\end{array}$} & $\begin{array}{l}\text { Instrumentación, } \\
\text { sin osteotomía }\end{array}$ \\
\hline 11 & \multicolumn{2}{|c|}{1} & 3 \\
\hline \multicolumn{4}{|c|}{ Vértebras superiores instrumentadas } \\
\hline T 10 & T 11 T 12 & L 1 & L 3 \\
\hline 1 & 3 & 1 & 2 \\
\hline
\end{tabular}

\section{Resultados}

De un total de 18 pacientes se excluyeron tres por no poder completar la evaluación de ODI posquirúrgica. En los 15 que quedaron, la edad media fue de 63.5 años (rango: 53-67), con una desviación estándar de 4.8 años. El sexo, el diagnóstico preoperatorio y la comorbilidad de los pacientes se muestran en la tabla 1.
Tabla 3. Diferencias en los resultados de las evaluaciones prequirúrgicas y posquirúrgicas del dolor y la funcionalidad

\begin{tabular}{|c|c|c|c|c|c|c|}
\hline \multicolumn{7}{|c|}{ Escala visual análoga del dolor (EVA) } \\
\hline & Media & Mínimo & Máximo & $\mathrm{DE}$ & t pareada & p \\
\hline Prequirúrgico & 8 & 7 & 9 & 0.65 & 18.89 & 0.011 \\
\hline Posquirúrgico & 3.53 & 3 & 5 & 0.74 & & \\
\hline \multicolumn{7}{|c|}{$\begin{array}{l}\text { Oswestry disability } \\
\text { Index (ODI) }\end{array}$} \\
\hline Prequirúrgico & 66.6 & 48 & 82 & 9.04 & 8.9576 & 0.017 \\
\hline Posquirúrgico & 31.6 & 0 & 56 & 15.19 & & \\
\hline
\end{tabular}

La estancia hospitalaria media fue de 3.9 días (rango: 3-8), con una desviación estándar de 1.3 días.

Todos los pacientes fueron manejados quirúrgicamente, con instrumentación de la columna y ampliación del canal espinal; la correlación clínico-radiográfica de los síntomas determinó el nivel para realizar la descompresión. El segmento que más requirió ampliación del canal espinal fue el L3L4 en un $53 \%$. Los detalles del tiempo quirúrgico, la cantidad de sangrado y la cirugía realizada (tipo de osteotomía y número de vértebras instrumentadas) se muestran en la tabla 2.

Las puntuaciones ODI y EVA antes y después de la cirugía tuvieron diferencias estadísticamente significativas (Figs. 1 y 2). En 13 de los 15 pacientes incluidos en el estudio hubo una diferencia de más de 15 puntos entre los valores preoperatorios y posoperatorios del ODI ( $p=0.017)$. También la evaluación del dolor con la EVA mostró una mejoría significativa de los valores posquirúrgicos comparados con los prequirúrgicos $(p=0.011)$. Los detalles se muestran en la tabla 3. El agrupamiento de los pacientes por tiempo de evolución postquirúrgica y las diferencias en la funcionalidad en cada grupo se muestran en la figura 3 (Fig. 3).

Entre las complicaciones reportadas, 3 (20\%) pacientes tuvieron lesiones durales y $1(6.7 \%)$ tuvo parestesias leves en los dermatomas correspondientes a L1; no se reportaron eventos tromboembólicos.

\section{Discusión}

La deformidad espinal del adulto es un trastorno espinal desafiante que se asocia con una gama amplia de presentaciones clínicas ${ }^{1}$. La cirugía representa un reto, ya que es compleja y los resultados a veces no son los esperados ${ }^{2,18}$. El nivel de investigación en 


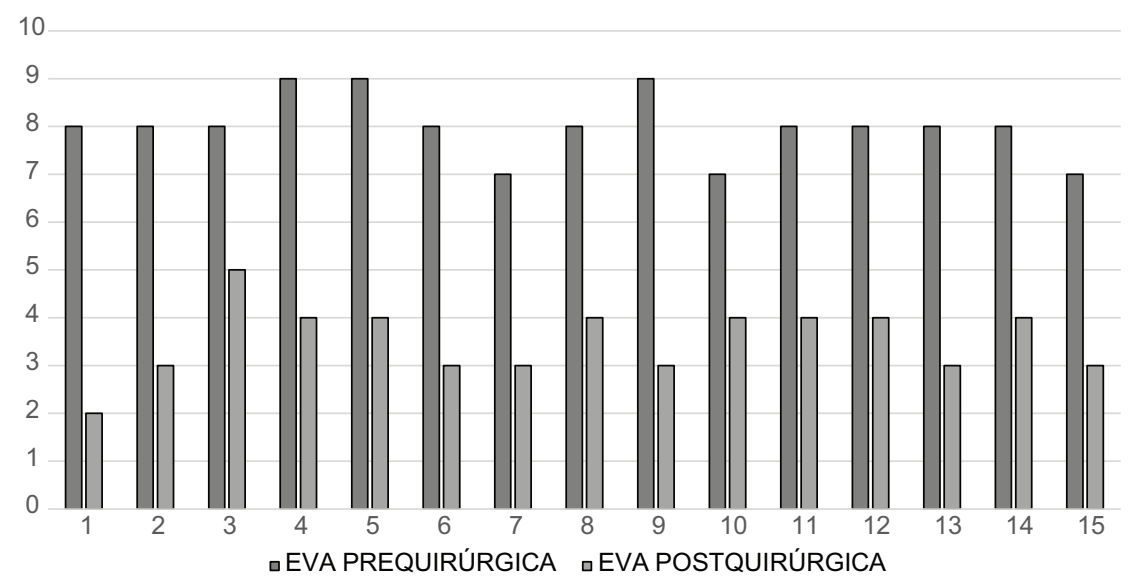

Figura 1. Comparación entre las puntuaciones de la escala visual análoga del dolor (EVA) antes y después de la cirugía.

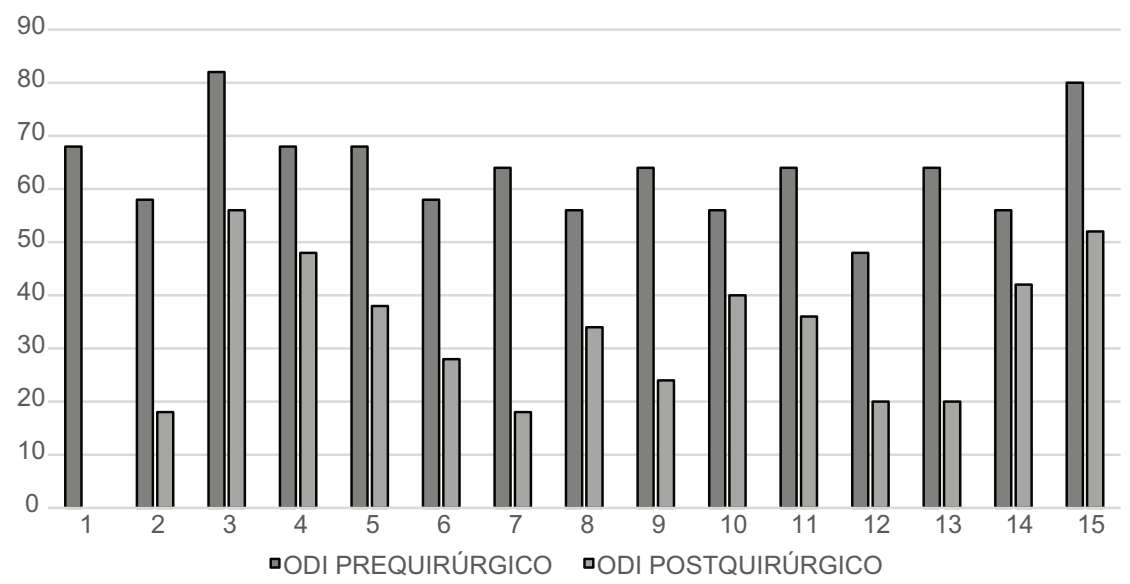

Figura 2. Comparación entre las puntuaciones del Oswestry Disability Index (ODI) antes y después de la cirugía. Destaca el paciente 1, con un puntaje posquirúrgico de 0 a los 6 meses de la cirugía.

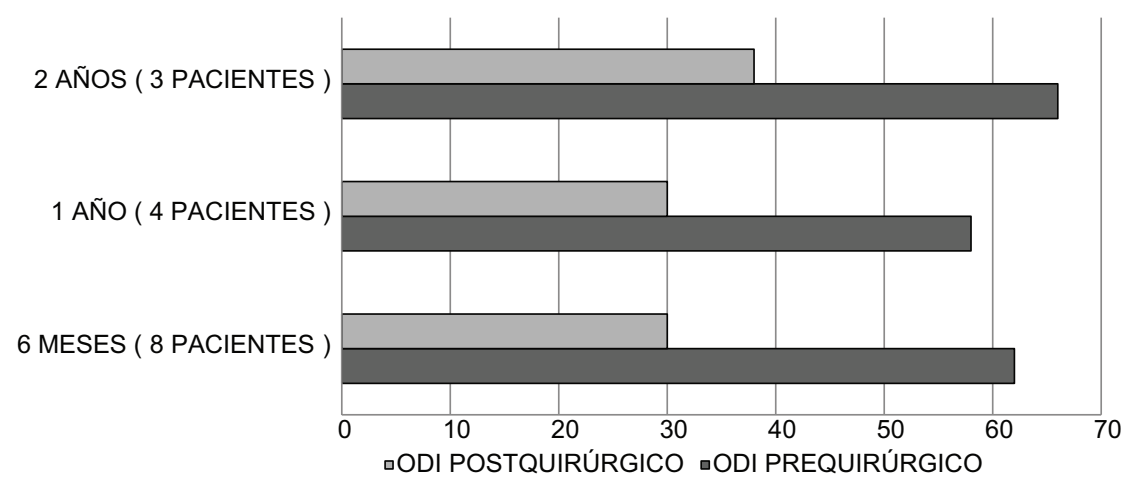

Figura 3. Comparación entre las puntuaciones del Oswestry Disability Index (ODI) antes y después de la cirugía, agrupadas por tiempo de evolución.

este campo aún no logra identificar cuál es el procedimiento quirúrgico que ofrece mejores resultados funcionales y una mayor mejoría del dolor².
Como primera línea de tratamiento se debe optar por el manejo médico. El tratamiento quirúrgico se tendrá en cuenta cuando fracasa el tratamiento 
médico. La literatura consultada refleja que los resultados del tratamiento quirúrgico superan a los del tratamiento médico ${ }^{19,20}$.

La finalidad del tratamiento debe ser disminuir la compresión neural, corregir el desequilibrio sagital o coronal, y fusionar la columna para lograr mejorar el dolor con una estabilidad en la columna vertebral ${ }^{19}$.

El aumento en la perspectiva de vida de la población ha hecho que esta patología se reconozca como un problema de salud pública importante.

En la literatura mexicana no existen reportes similares de pacientes con patología degenerativa de columna vertebral, por lo que resulta difícil comparar nuestros resultados con los de otros estudios realizados en México. Las características de esta población coinciden con las de otros estudios en cuanto a edad de presentación, sexo y limitación funcional ${ }^{14}$.

Un aspecto que se debe considerar es que datos como el sangrado y el tiempo quirúrgico están en relación con las aptitudes propias del cirujano y con la complejidad de cada paciente. Sin embargo, mientras que Elgafy, et al. ${ }^{21}$ reportaron un sangrado de 650 a $2839 \mathrm{ml}$, en este trabajo las cifras son superiores, ya que van desde 1000 hasta $3500 \mathrm{ml}$ (media $=1940 \pm$ $841 \mathrm{ml}$ ), y nuestros resultados finales fueron muy positivos.

La cirugía para la deformidad de la columna vertebral, como toda intervención quirúrgica de columna, no está exenta de riesgos. La incidencia de eventos tromboembólicos se ha reportado hasta del $4.3 \%$, con una tasa de mortalidad alta $^{18}$, considerada como una complicación quirúrgica fata|20,22-24. En este estudio no ocurrió esta complicación, aunque se reportaron complicaciones menores. Cabe mencionar que el tiempo quirúrgico prolongado, considerado así al mayor de 8 horas, incrementa el riesgo para desarrollar complicaciones $^{9}$. En el presente trabajo se reporta un tiempo medio menor ( 6.5 horas aproximadamente; solo en dos pacientes fue de 8 horas).

Los pacientes con este tipo de alteraciones tienden a disminuir los puntajes en las escalas de evaluación de la capacidad funcional. Los pacientes con discapacidad moderada experimentan dolor y dificultad para sentarse, levantar objetos y pararse. Los viajes y la vida social son difíciles, y no pueden trabajar. El cuidado personal, la actividad sexual y el sueño no se ven afectados, y mejoran el dolor con analgésicos ${ }^{11}$.

Teles, et al..$^{22}$, en el año 2017, presentaron informes de mejoría de la discapacidad de $19.1 \pm 9$ puntos en el ODI, y Daubs, et al. ${ }^{23}$, en el año 2007, de 24 puntos. En el presente trabajo, la mejoría de puntuación del
ODI posquirúrgico comparado con el prequirúrgico fue de $32 \pm 13$ puntos, mucho mayor que en las referencias internacionales.

También se debe considerar que la mejoría en estos pacientes del dolor posquirúrgico comparado con el prequirúrgico (EVA $4.46 \pm 0.91$ puntos) tuvo una diferencia estadísticamente significativa. Todo ello concuerda con la literatura internacional, que afirma que el tratamiento quirúrgico ofrece resultados buenos en funcionalidad y dolor referido por los pacientes ${ }^{7}$.

Yoshida, et al. ${ }^{24}$ reportan también mejoría global y en todos los dominios a los 2 años de la cirugía al compararlos con el preoperatorio, pero "dolor», «marcha» y «pararse» registraron un empeoramiento al año y a los 5 años.

Llaman la atención los estudios en población latinoamericana utilizando también el ODI, con reporte de un $56 \%$ de pacientes con limitación funcional mínima y un $6 \%$ de discapacidad a los 6 meses de la cirugía $a^{14,15}$. Nuestros resultados sugieren que la mejoría en la funcionalidad persiste a través del tiempo (Fig. 3). Nosotros consideramos que el seguimiento de estos pacientes debe continuarse hasta los 5 años para poder determinar el comportamiento de la funcionalidad y del dolor.

Finalmente, hay que recordar que en la patología de columna degenerativa los procedimientos quirúrgicos no son curativos; en todos se registra cierto grado de discapacidad residual, pero el objetivo es mejorar la funcionalidad y la calidad de vida del paciente.

Podemos concluir que los pacientes manejados quirúrgicamente por deformidad espinal en nuestra unidad presentan mejoría tanto de la funcionalidad como del dolor a 1 año de seguimiento. Sugerimos que el seguimiento de estos pacientes se realice a 5 años para determinar los resultados en ese tiempo.

\section{Conflicto de intereses}

Los autores no declaran conflictos de intereses.

\section{Financiamiento}

Los autores no recibieron ayuda ni financiación para el presente trabajo.

\section{Responsabilidades éticas}

Protección de personas y animales. Los autores declaran que para esta investigación no se han realizado experimentos en seres humanos ni en animales. 
Confidencialidad de los datos. Los autores declaran que han seguido los protocolos de su centro de trabajo sobre la publicación de datos de pacientes.

Derecho a la privacidad y consentimiento informado. Los autores han obtenido el consentimiento informado de los pacientes y/o sujetos referidos en el artículo. Este documento obra en poder del autor de correspondencia.

\section{Bibliografía}

1. Kim YJ, Hyun SJ, Cheh G, Cho S KC, Rhim SC. Decision making algorithm for adult spinal deformity surgery. J Korean Neurosurg Soc. 2016;59:327-33.

2. Acaroglu E, European Spine Study Group. Decision-making in the treatment of adult spinal deformity. EFORT Open Rev. 2016;1:167-76.

3. Bess S, Line B, Fu K-M, McCarthy I, Lafage V, Schwab F, et al. The health impact of symptomatic adult spinal deformity: comparison of deformity types to united states population norms and chronic diseases. Spine. 2016;41:224-33.

4. Huang L-C, Chung W-F, Liu S-W, Chang P-Y, Chen L-F, Wu J-C, et al. Lower risk of stroke after deformity surgery: long term benefit demonstrated by a national cohort study. Int $\mathrm{J}$ Environ Res Public Health. 2015;12:12618-27.

5. Fu X, Sun X-L, Harris JA, Sheng S-R, Xu H-Z, Chi Y-L, et al. Long fusion correction of degenerative adult spinal deformity and the selection of the upper or lower thoracic region as the site of proximal instrumentation: a systematic review and meta-analysis. BMJ Open. 2016;6:e012103.

6. Bokshan SL, Godzik J, Dalton J, Jaffe J, Lenke LG, Kelly MP. Reliability of the Revised Scoliosis Research Society and Oswestry Disability Index (Odi) questionnaires in adult spinal deformity when administered by telephone. Spine J. 2016;16:1042-6.

7. Good CR, Auerbach JD, O'Leary PT, Schuler TC. Adult spine deformity. Curr Rev Musculoskelet Med. 2011;4:159-67.

8. Smith JS, Shaffrey CI, Glassman SD, Carreon LY, Schwab FJ, Lafage V, et al. Clinical and radiographic parameters that distinguish between the best and worst outcomes of scoliosis surgery for adults. Eur Spine J. 2013;22:402-10.

9. De la Garza-Ramos R, Nakhla J, Gelfand Y, Echt M, Scoco AN, Kinon MD, et al. Predicting critical care unit-level complications after long-segment fusion procedures for adult spinal deformity. J Spine Surg. 2018;4:55-61.

10. Lee NJ, Shin JI, Kothari P, Kim JS, Leven DM, Steinberger J, et al. Incidence, impact, and risk factors for 30-day wound complications following elective adult spinal deformity surgery. Global Spine Journal. 2017;7:417-24.
11. Fairbank JC, Pynsent PB. The Oswestry Disability Index. Spine. 2000;25:2940-52.

12. Laratta JL, Ha A, Shillingford JN, Makhni MC, Lombardi JM, Thuet E, et al. Neuromonitoring in spinal deformity surgery: a multimodality approach. Global Spine Journal. 2018;8:68-77.

13. Sirvanci M, Bhatia M, Ganiyusufoglu KA, Duran C, Tezer M, Azturk C, et al. Degenerative lumbar spinal stenosis: correlation with Oswestry Disability Index and MR Imaging. Eur Spine J. 2008;17:679-85.

14. Uruchi-Limachi DM, Sea-Aramayo JM. Evaluación funcional mediante la escala de Oswestry en pacientes con artrodesis postero-lateral por canal lumbar estrecho. Rev Med La Paz. 2017;23:6-12.

15. Terran J, McHugh BJ, Fischer CR, Lonner B, Warren D, Glassman S, et al. Surgical treatment for adult spinal deformity: projected cost effectiveness at 5 year follow-up. Ocshner Journal. 2014;14:14-22.

16. Mendoza-Chávez SR, Corpus-Mariscal E, Aguilar-Madrid G. Adaptación transcultural del cuestionario "Oswestry Disability Index 2.0" para la evaluación de incapacidad funcional de pacientes con dolor lumbar en México. [Tesis]. México: División de Estudios de Postgrado e Investigación, Universidad Nacional Autónoma de México, Facultad de Medicina; 2008. (Consultado el 19 de marzo de 2019.) Disponible en: http://oreon. dgbiblio.unam.mx/F/IF9IH3RDBCIEGDT6H6EN63I3S1VNSRF8YV6XK8MUB61A31FQ74-15185?func=full-set-set\&set_number=014805\&set_ entry $=000002 \&$ format $=999$.

17. Diebo B, Liu S, Lafage V, Schwab F. Osteotomies in the treatment of spinal deformities: indications, classification and surgical planning. Eur $\mathrm{J}$ Orthop Surg Traumatol. 2014;24:S11-S20.

18. Iyer S, Klineberg EO, Zebala LP, Kelly MP, Hart RA, Gupta MC, et al. Dural tears in adult deformity surgery: incidence, risk factors, and outcomes. Global Spine Journal. 2018;8:25-31.

19. Merrill RK, Kim JS, McNeill I, Overley SC, Dowdell JE, Caridi JM, et al. Negative sagittal balance following adult spinal deformity surgery. Global Spine Journal. 2018;8:149-55.

20. Kim HJ, lyer S, Diebo BG, Kelly MP, Sciubba D, Schwab F, et al. Clinically significant thromboembolic disease in adult spinal deformity surgery: incidence and risk factors in 737 patients. Global Spine Journal. 2018;8:224-30.

21. Elgafy H, Bransford RJ, McGuire RA, Dettori JR, Fisher D. Blood loss in major spine surgery. Are there effective measures to decrease massive hemorrhage in major spine fusion surgery? Spine. 2010;35:S47-S56.

22. Teles AR, Mattei TA, Righesso O, Falavigna A. Effectiveness of operative and nonoperative care for adult spinal deformity: systematic review of the literature. Global Spine Journal. 2017;7:170-8.

23. Daubs MD, Lenke LG, Cheh G, Stobbs G, Bridwell KH. Adult spinal deformity surgery. Complications and outcomes in patients over age 60 . Spine. 2007;32:2238-44.

24. Yoshida G, Hasegawa T, Yamato Y, Kobayashi S, Shin O, Banno T, et al. Minimum clinically important differences in Oswestry Disability Index domains and their impact on adult spinal deformity surgery. Asian Spine Journal. 2019;13:35-44. 